\title{
Protective effect of Toxocara vitulorum extract against alpha- naphthylisothiocyanate-induced cholangitis in rat
}

Abeer Mahmoud Badr*, Mohamed Farid, Ahmed Abdel Aziz Biomy, Ayman Saber Mohamed, Noha Ahmed Mahana and Sohair Ramadan Fahmy

\begin{abstract}
Background: Cholestasis is the major cause of bile acid accumulation leading to liver damage. Chronic infection of worms can modulate the immune response towards T helper (Th)2-related cytokines. The present study aims to evaluate the protective impact of an ascarid nematode Toxocara vitulorum extract (TVE) against alpha-naphthylisothiocyanate (ANIT)induced cholangitis male wistar rat model compared to ursodeoxycholic acid (UDCA) as a standard drug.

Results: Pretreatment with TVE and/or UDCA induced a marked reduction in the levels of liver function tests and malondialdehyde, while antioxidant markers were increased compared to cholestatic rats. Pretreatment with either TVE or combination before cholangitis induction attenuated the predominant Th1-related cytokines (IFN- $y$ and TNF-a) to Th2 (IL-13 and IL-10). TVE administration promoted higher expression levels of BCl-2 protein and lower levels of caspase-3 compared to cholestatic rats.

Conclusions: Treatment with TVE has improved the liver functions and elevated the levels of oxidative stress markers. The upregulation of Th2-related cytokines and suppression of apoptosis through caspase-3 might be considered as a potential mechanism of TVE. Thereby, this natural extract revealed an opportunity for use in treatment of cholangitis disease.
\end{abstract}

Keywords: Toxocara vitulorum, Cholestasis, T helper immune responses, Oxidative stress markers, Apoptosis

\section{Background}

Liver cholestasis is a devastating liver condition defined as an impairment of bile flow that leads to toxic bile acid accumulation in hepatocytes (Li et al., 2019). It results in hepatocellular damage followed by inflammation, fibrosis, and liver cirrhosis (Jin et al., 2013). There are numerous distinctive fundamental causes in this condition, including inherited and acquired pathologies (Alkhedaide, Ismail, Alotaibi, Nassan, \& Shehri, 2018).

During cholestatic liver disease, evidence over the last decade has indicated that bile acids may injure liver cells by initiating a cytokine-mediated inflammatory response

\footnotetext{
* Correspondence: abeerbadr@gmail.com

Zoology Department, Faculty of Science, Cairo University, Giza 12316, Egypt
}

in addition to their direct toxic effects as a detergent (Woolbright, Antoine, Jenkins, Bajt, \& Park, 2013). Cholestasis is characterized by bile acid accumulation, leads to inflammation, necrosis, and apoptosis in hepatocytes (Dilger et al., 2012). Regulation of apoptosis through the intrinsic pathway of mitochondria and is characterized by the release of mitochondrial intermembrane space proteins, including cytochrome c, apoptosis-inducing factor, a second mitochondrial activator of caspases into the cytosol (van Loo et al., 2002). Cytosolic cytochrome c subsequently activates a multiprotein complex referred to as the apoptosome, which in turn leads to cleavage of procaspase-9 and downstream effector caspases (e.g., caspase-3), resulting in cell death. The antiapoptotic Bcl2 proteins inhibit apoptosis through the inhibition of the 
proapoptotic $\mathrm{Bcl}-2$ proteins, $\mathrm{Bcl}-2$-associated $\mathrm{X}$ protein (BAX), and Bcl-2 homologous antagonist killer (BAK) (Zaman, Wang, \& Gandh, 2014).

The liver is a central immunological organ with a high exposure to circulating antigens and endotoxins from the gut microbiota (Heymann \& Tacke, 2013). Research evidences demonstrate that an impairment of normal bile flow and excessive accumulation of potentially toxic bile acids are considered the most important conditions that induced autoimmune cholestatic liver diseases, including primary biliary cholangitis (PBC) and primary sclerosing cholangitis (PSC) (Terziroli et al., 2017). During $\mathrm{PBC}$, patients develop a well-orchestrated immune reaction, both innate and adaptive, against mitochondrial antigens that specifically targets intrahepatic biliary cells (Lleo, Marzorati, Anaya, \& Gershwin, 2017).

It has been reported that intrahepatic cholestasis can adequately mimic in rat ( $\mathrm{Li}$ et al., 2019). Alphanaphthylisothiocyanate (ANIT) has widely used chemical to induce cholestasis in experimental animals (Hua et al., 2019). Liver injury, induced by ANIT, occurred mainly via the formation of radical species and lipid peroxidation (Ohta, Kongo, Sasaki, \& Harada, 1999), and infiltration of neutrophils via a CD18-dependent mechanism (Kodali, Wu, Lahiji, Brown, \& Maher, 2006).

The CD4+ T cell subsets are functionally participated in altered cytokine environments, being a central issue in autoimmune and immune-mediated diseases (Smits et al., 2001), $T$ (helper) h1 and Th2 cells are involved in cellular immunity, classified according to their secreted cytokines. The immune responses of Th1 cells are produced against bacteria and virus infection, while Th2 cells contribute to protective immunity against extracellular pathogens and parasitic infections (Abe, Hiasa, \& Onji, 2013). In response to inflammatory insults, the inhabitant intrahepatic $\mathrm{T}$ cells play a unique role in maintaining the balance between tolerance and immunity (Crispe, 2009).The equilibrium between proinflammatory state of Th1 and the defensive actions of Th2 can determine the net result of autoimmune diseases. Th17 cells are believed to play an important role in the development of a variety of autoimmune diseases, including autoimmune liver disease (Fouser, Wright, Dunussi-Joannopoulos, \& Collins, 2008).

Currently, the only drug available for PBC treatment is ursodeoxycholic acid (UDCA). However, it is limited only in the early stage of PBC (He, Mennone, Boyer, \& Cai, 2011). Therefore, the search for novel therapeutic compounds is still needed. Helminths infections and allergy highly induce Th2 immune response (Erb, 2007), which can protect an individual from allergic condition and developing lethal immunopathology (Goddey, Osagie, \& Maliki, 2010). It was found that nematode infections may induce either severe immunosuppression or enhancement of the Th2 responses (Caraballo \&
Acevedo, 2011). Chronic infection of worms can modulate the immune response towards Th2-related cytokines, including interleukin (IL)-13. Thereby, the current study aims to investigate the immunomodulatory role of an ascarid nematode, Toxocara (T.) vitulorum extract (TvE), on the polarization of Th cells and liver histopathology in the rat cholangitis model as well as clarified the likelihood view of its use as a treatment option for cholestasis.

\section{Methods}

\section{Chemicals and reagents}

Alpha-naphthylisothiocyanate (ANIT) purchased from Sigma-Aldrich (St. Louis, MO, USA). Ursofalk (ursodeoxycholic acid) (UDCA) obtained from UDCA capsule was got from Minapharm Pharmaceutical, Egypt. Kits for all biochemical parameters and Tris- $\mathrm{HCl}$ buffer $(\mathrm{pH} 7.4)$ were purchased from Biodiagnostic Company (El Moror St., Dokki, Egypt). Levels of IFN- $\gamma$ (eBioscience, USA), IL10 (eBioscience, USA), and IL-13 (Novus Biologicals, USA) were quantified by enzyme-linked immunosorbent assay (ELISA). TNF- $\alpha$ and IL-17A were measured by indirect ELISA MAX ${ }^{\mathrm{TM}}$ Deluxe Sets (BioLegend, Inc., San Diego, CA, USA). Hematoxylin and eosin as well as Masson's Trichrome stain were purchased from SigmaAldrich (St. Louis, MO, USA).

\section{Toxocara vitulorum study Toxocara vitulorum collection}

Adult worms of T. vitulorum were collected from the small intestines of naturally infected young buffalo calves, freshly slaughtered at a local abattoir in Giza Province, Egypt. The worms were transported in a flask containing Goodwin's solution to the laboratory.

\section{Toxocara vitulorum extract (TvE) preparation}

The extract of adult $T$. vitulorum worms were performed according to (Souza, Faquim-Mauro, and Macedo, 2002). Briefly, live adult worms washed several times in saline and homogenized in borate buffered saline (BBS) at $\mathrm{pH}$ 8.0. The homogenate was centrifuged at $10,000 \mathrm{rpm}$ for 1 $h$. Then, the precipitate was resuspended and stirred in $400 \mathrm{ml} \mathrm{BBS}$ overnight at $4{ }^{\circ} \mathrm{C}$. The suspension was centrifuged, the supernatant was dialyzed against distilled water overnight at $4{ }^{\circ} \mathrm{C}$, and then centrifuged at $10,000 \mathrm{rpm}$ for $2 \mathrm{~h}$ and lyophilized. TvE aliquots were diluted in sterile $0.01 \mathrm{M}$ sodium phosphate-buffered saline (PBS) at pH 7.2.

\section{Animals}

\section{Ethical consideration}

Experimental protocols and procedures used in this study were approved by the Cairo University-Institutional Animal Care and Use Committee (CU-IACUC) (Egypt), (approval no. CU/I/F/95/17). The subject animals were outbred male 
Wistar (outbred) strained, weighing $150 \pm 5$ g. (National Research Center, Dokki, Egypt). During the period of the acclimation (1 week), the rats were fed standard rodent food pellets (Agricultural, Industrial Integration Company, Giza, Egypt) and provided with tap water ad libitum. The room was maintained under a 12:12 h light:dark schedule with the white light on between 02:00 and 14:00 $\mathrm{h}$ and continuous dim red light (two 60-W bulbs, Serma Electrical, Cairo, Egypt) enabling observation during the dark period, at a constant temperature $\left(22-25^{\circ} \mathrm{C}\right)$. Rats were grouped and housed in polyacrylic cages (six animals per cage) and supplied with bedding (saw dust) and nesting (Kleenex tissues) material.

\section{Induction of cholangitis}

This selected species of rats is appropriate for cholangitis that mimic human disease. Cholangitis was induced via the intragastric administration of rats with a single oral dose of ANIT (100 mg/kg, in olive oil) (Sugiura et al., 2018).

\section{Experimental design}

Thirty-six rats were randomly allocated into five groups as follows:

Group 1 (control, 6 rats/group): rats of this group were administered orally with olive oil, the vehicle of ANIT, and injected I.P. with $0.01 \mathrm{M}$ PBS, the vehicle of TvE. Group 2 (TvE, 6 rats/group): rats of this group injected intraperitoneally (I.P.) with a single dosage of 1 $\mathrm{mg} / \mathrm{rat} \mathrm{TvE}$ as described by (Rocha et al., 2008). Group 3 (ANIT, 24 rats/group): this group subdivided into four subgroups (6 rats/group) as follows: subgroup I: (ANIT subgroup): rats of this subgroup injected I.P. with olive oil 30 min prior to ANIT injection. Subgroup II (ANIT $+\mathrm{TvE}$ subgroup): rats injected I.P. with a single dosage of $1 \mathrm{mg} / \mathrm{rat}$ of TvE $30 \mathrm{~min}$ prior to ANIT injection. Subgroup III (ANIT+ UDCA): rats of this subgroup injected with single dosage of UDCA ( $80 \mathrm{mg} / \mathrm{kg}$ body weight) 30 min prior to ANIT injection. Subgroup IV (ANIT + TvE + UDCA): rats injected with single dosage of both $\mathrm{TvE}$ and UDCA $30 \mathrm{~min}$ prior to ANIT injection.

\section{Monitoring and handling}

The rats were monitored daily for any unexpected weight loss and change of normal behavior. The animals that suffered from any of the previous signs of pain and distress were euthanized (humane killing). All animals were euthanized after $48 \mathrm{~h}$ of ANIT administration under deep anesthesia with sodium pentobarbital and sacrificed by cardiac puncture. Blood was collected in centrifuge tubes. Liver was taken away and instantly blotted using filter paper to remove traces of blood and then divided into two parts: the first part stored at -80 ${ }^{\circ} \mathrm{C}$ for biochemical studies, while the second part was suspended in $10 \%$ neutral buffer formalin solution for fixation preparatory to histopathological processing.

\section{Liver homogenate preparation}

Liver tissue samples were homogenized $(10 \% \mathrm{w} / \mathrm{v})$ in ice-cold 0.1 M Tris- $\mathrm{HCl}$ buffer ( $\mathrm{pH}$ 7.4). The homogenate was centrifuged at $3000 \mathrm{rpm}$ for $15 \mathrm{~min}$ at $4{ }^{\circ} \mathrm{C}$ and the resultant supernatant was used for biochemical analysis.

\section{Serum biomarkers for liver function tests}

Total protein estimated by the method of Tietz, albumin, aspartate aminotransferase (AST) and alanine aminotransferase (ALT), alkaline phosphatase (ALP), $\gamma$ glutamyltransferase (GGT), and bilirubin were determined according to the manufacturer's instructions using Spectrum Diagnostics and Bio-diagnostic kits (Giza, Egypt).

\section{Oxidative stress markers assessment}

Oxidative stress markers were measured in the resultant supernatant of liver homogenate. The appropriate kits were used for the determination of malondialdehyde (MDA) level, glutathione reduced (GSH), and catalase (CAT).

\section{Cytokines assays}

The serum levels of IFN- $\gamma$ (R\&D Systems, Inc., USA), L13 (EMELCA Bioscience), TNF- $\alpha$, and IL-10 (Koma Biotech, Inc., Korea) as well as IL-17 (Biolegend, MAXTM Deluxe, Inc., USA) were quantified by ELISA kits according to the producer instructions. The optical density was read at $450 \mathrm{~nm}$ by a microplate reader (Das, Italy). The cytokine concentration was calculated from the standard curve and expressed as picogram/milliliter.

\section{Histopathological examination}

The preserved liver tissue samples were washed in tap water, dehydrated through an upgraded series of ethanol (50, 70, 80, 90, and followed by absolute ethanol), cleared in xylene, and then embedded in paraffin. The paraffin-embedded samples were cut into $4 \mu \mathrm{m}$ sections, which were then routinely stained with hematoxylin and eosin. In Masson's Trichrome method, the liver sections were deparaffinized and rehydrated through descending series of alcohols and then stained in Biebrich scarletacid fuchsin solution. Then, sections were differentiated in phosphomolybdic-phosphotungstic acid solution. Sections were transferred directly to aniline blue solution and differentiated in 1\% acetic acid solution, dehydrated very quickly through an ascending series of alcohols, cleared in xylene, and mounted with the resinous mounting medium. 


\section{Immunohistochemical analysis}

By using avidin-biotin-peroxidase method, the paraffin embedded samples were cut into $3 \mu \mathrm{m}$ sections and mounted on positively charged slides for caspase- 3 and Bcl-2 immunohistochemical examination. Sections were dewaxed, rehydrated, and autoclaved at $95{ }^{\circ} \mathrm{C}$ for $20 \mathrm{~min}$ in antigen recovery buffer (10 $\mathrm{mM}$ citrate buffer, $\mathrm{pH}$ 6). After washing with phosphate-buffered saline (PBS), endogenous peroxidase was blocked using 3\% $\mathrm{H} 2 \mathrm{O} 2$ in methanol for $15 \mathrm{~min}$. A primary rat-specific antibody for caspase-3 and Bcl-2 (cat. no. RB 1197 B0, B1; Thermo Fisher Scientific Inc.: IgG1k; DAKO Corp. A/S, Glostrup, Denmark) was added following dilution in PBS (1:100) and incubated for $30 \mathrm{~min}$. Then the slides were washed three times for $3 \mathrm{~min}$ each with PBS. Subsequently, horseradish peroxidase conjugated goat anti-mouse IgG secondary antibodies (cat. no. 32230; Thermo Fisher Scientific Inc.) was applied to the tissue sections and incubated for $30 \mathrm{~min}$. The slides were washed three times for 3 min each with PBS, and then visualized by adding metal enhanced DAB substrate working solution (Thermo Fisher Scientific Inc.) to the tissues and incubating for $10 \mathrm{~min}$. Next, the slides were washed two times with PBS (3 min each time) and then counter stained by adding an adequate amount of hematoxylin to the slide to cover the entire tissue surface. The examiners, blinded to the experimental groups, counted the cells labeled with caspase- 3 and Bcl-2 throughout five random lesion regions in the stained areas under a 100 light microscope. Then, the expression levels of caspase3 and $\mathrm{Bcl}-2$ were analyzed by mean integrated optical density (IOD).

\section{Statistical analysis}

Values were expressed as means \pm standard error (SE). To evaluate differences between the groups studied, one-way analysis of variance (ANOVA) with the Duncan post hoc test was used to compare the group means and $P<0.05$ was considered statistically significant. SPSS, for Windows (version 20.0) was used for the statistical analysis.

\section{Results}

\section{Physiological parameters}

Enzyme activities of ALT, AST, ALP, GGT, and concentrations of total bilirubin in ANIT and TvE groups showed a significant increase $(P<0.05)$ as compared to control group. While total proteins and albumin showed non-significant $(P>0.05)$ change (Table 1$)$. On the other hand, the treatments with TvE and/or UDCA caused a significant $(P<0.05)$ decrease in activities of AST, ALT, ALP, bilirubin, and GGT compared with ANIT group. Significant decreases in GSH and CAT levels were observed in ANIT group as compared to control group.
However, MDA concentration was increased significantly. After treatment with TvE and/or UDCA, there is a significant $(P<0.05)$ increase in GSH and CAT levels as compared to ANIT group, while MDA concentration decreased significantly.

\section{Immunological cytokines \\ $T$ helper-1-related cytokines}

IFN- $\gamma$ is one of characteristic cytokines related to Th1immune responses. Administration of TvE (TvE group) induced a decrease in the serum levels of IFN- $\gamma$ and TNF$\alpha$ but did not reach significant differences compared to ANIT rats (Fig. 1a, b). Treatment with TvE before ANIT administration promoted a significant decrease $(P<0.05)$ in the levels of both IFN- $\gamma$ and TNF- $\alpha$ compared to ANIT group of rats. Also, rats treated with UDCA before ANIT (ANIT + UDCA group) showed a significant reduction in the levels of IFN- $\gamma$ and TNF- $\alpha$ serum levels when compared with ANIT group of rats. Combination treatment of TvE and UDCA before ANIT-treated rats induced significant lower levels $(P<0.05)$ of IFN- $\gamma$ and TNF- $\alpha$ than those in ANIT group.

\section{$T$ helper-2-related cytokines}

Induction of cholangitis rat model by ANIT administration led to a significant decrease $(P<0.05)$ in the levels of IL-13 but has no significant effect on IL-10 compared to control group. On the other hand, the levels of IL-13 and IL-10 significantly increased $(P<0.05)$ in treated rats with TvE before ANIT administration (TvE + ANIT group) compared to ANIT group. Rats administered with UDCA before ANIT treatment showed significantly higher levels $(P<0.05)$ of IL-13 than in ANIT group. The combination of TvE + UDCA administration before ANIT treatment can promote significantly higher levels $(P<0.05)$ of both IL-10 and IL-13 than in ANIT group (Fig. 1c, d).

\section{$T$ helper-17-related cytokine}

ANIT-treated rats showed a slight increase in the level of IL-17A compared to control rats, but administration of either TvE or UDCA showed a little decrease in IL17A levels compared to ANIT group. Interestingly, rats administered with the combination of TvE + UDCA before ANIT administration caused a significant increase $(P<0.05)$ in the levels of IL-17A (Fig. 1e).

\section{Histological evaluation}

Liver of control and TvE rats showed a normal architecture, with hepatic lobules around the central vein and each lobule consisting of hepatic cords of hepatocytes (Fig. 2a, b). Liver of ANIT rats showed severe congestion of central vein and necrosis with cytoplasmic vacuolization of centrolobular hepatocytes (Fig. 2c). Cholestasis is 
Table 1 Effect of Toxocara vitulorum extract (TVE) on biochemical parameters of cholestatic rats

\begin{tabular}{|c|c|c|c|c|c|c|c|c|c|c|}
\hline Groups & $\begin{array}{l}\text { AST (U/ } \\
\mathrm{ml})\end{array}$ & $\begin{array}{l}\text { ALT (U/ } \\
\mathrm{ml})\end{array}$ & $\begin{array}{l}\text { GGT (U/ } \\
\text { L) }\end{array}$ & $\begin{array}{l}\text { ALP (U/ } \\
\text { L) }\end{array}$ & $\begin{array}{l}\text { Total protein } \\
\text { (g/dl) }\end{array}$ & $\begin{array}{l}\text { Albumin } \\
\text { (g/dl) }\end{array}$ & $\begin{array}{l}\text { Bilirubin } \\
(\mathrm{mg} / \mathrm{dl})\end{array}$ & $\begin{array}{l}\text { MDA (nmol/g } \\
\text { tissue) }\end{array}$ & $\begin{array}{l}\mathrm{GSH}(\mathrm{mg} / \mathrm{g} \\
\text { protein) }\end{array}$ & $\begin{array}{l}\text { CAT (U/g } \\
\text { protein) }\end{array}$ \\
\hline Control & $\begin{array}{l}10.04 \pm \\
0.79^{\mathrm{a}}\end{array}$ & $\begin{array}{l}11.68 \pm \\
0.59^{\mathrm{a}}\end{array}$ & $\begin{array}{l}5.79 \pm \\
2.41^{\mathrm{a}}\end{array}$ & $\begin{array}{l}10.85 \pm \\
0.72^{\mathrm{a}}\end{array}$ & $7.65 \pm 0.37^{a}$ & $\begin{array}{l}3.22 \pm \\
0.09^{\mathrm{a}}\end{array}$ & $1.14 \pm 0.29^{\mathrm{a}}$ & $1.67 \pm 0.07^{a}$ & $17.11 \pm 0.08^{c}$ & $1.77 \pm 0.09^{c}$ \\
\hline TvE & $\begin{array}{l}13.19 \pm \\
3.07^{\mathrm{b}}\end{array}$ & $\begin{array}{l}12.86 \pm \\
2.46^{\mathrm{b}}\end{array}$ & $\begin{array}{l}10.63 \pm \\
2.33^{b}\end{array}$ & $\begin{array}{l}15.37 \pm \\
1.11^{\mathrm{b}}\end{array}$ & $7.39 \pm 0.30^{a}$ & $\begin{array}{l}3.19 \pm \\
0.10^{\mathrm{a}}\end{array}$ & $61 \pm 0.23^{b}$ & $1.76 \pm 0.15^{\mathrm{a}}$ & $16.59 \pm 0.29^{c}$ & $1.57 \pm 0.02^{c}$ \\
\hline ANIT & $\begin{array}{l}21.21 \pm \\
1.45^{c}\end{array}$ & $\begin{array}{l}16.18 \pm \\
0.82^{c}\end{array}$ & $\begin{array}{l}30.52 \pm \\
1.37^{c}\end{array}$ & $\begin{array}{l}24.14 \pm \\
1.23^{c}\end{array}$ & $0.18^{\mathrm{a}}$ & $\begin{array}{l}3.28 \pm \\
0.14^{\mathrm{a}}\end{array}$ & $\pm 0.15^{c}$ & $4.47 \pm 0.11^{c}$ & $9.03 \pm 0.70^{a}$ & $0.76 \pm 0.03^{a}$ \\
\hline ANIT + TvE & $\begin{array}{l}12.15 \pm \\
0.44^{\mathrm{b}}\end{array}$ & $\begin{array}{l}12.97 \pm \\
0.66^{\mathrm{b}}\end{array}$ & $\begin{array}{l}14.45 \pm \\
0.80^{\mathrm{b}}\end{array}$ & $\begin{array}{l}14.48 \pm \\
0.93^{\mathrm{b}}\end{array}$ & $7.62 \pm 0.30^{\mathrm{a}}$ & $\begin{array}{l}2.93 \pm \\
0.13^{\mathrm{a}}\end{array}$ & $1.69 \pm 0.08^{b}$ & $2.12 \pm 0.07^{b}$ & $13.50 \pm 0.43^{b}$ & $1.23 \pm 0.02^{b}$ \\
\hline ANIT + UDCA & $\begin{array}{l}13.13 \pm \\
2.08^{\mathrm{b}}\end{array}$ & $\begin{array}{l}12.29 \pm \\
0.30^{\mathrm{b}}\end{array}$ & $\begin{array}{l}16.38 \pm \\
2.26^{\mathrm{b}}\end{array}$ & $\begin{array}{l}15.43 \pm \\
0.70^{\mathrm{b}}\end{array}$ & $7.05 \pm 0.12^{a}$ & $\begin{array}{l}3.04 \pm \\
0.10^{\mathrm{a}}\end{array}$ & $1.73 \pm 0.13^{b}$ & $2.82 \pm 0.21^{b}$ & $12.87 \pm 0.42^{b}$ & $1.09 \pm 0.05^{b}$ \\
\hline $\begin{array}{l}\text { ANIT + UDCA } \\
+ \text { TvE }\end{array}$ & $\begin{array}{l}13.14 \pm \\
0.84^{\mathrm{b}}\end{array}$ & $\begin{array}{l}13.63 \pm \\
0.37^{b}\end{array}$ & $\begin{array}{l}15.09 \pm \\
1.28^{\mathrm{b}}\end{array}$ & $\begin{array}{l}14.90 \pm \\
1.11^{\mathrm{b}}\end{array}$ & $8.15 \pm 0.18^{\mathrm{a}}$ & $\begin{array}{l}3.27 \pm \\
0.13^{\mathrm{a}}\end{array}$ & $1.77 \pm 0.21^{b}$ & $2.53 \pm 0.10^{b}$ & $12.90 \pm 0.08^{\mathrm{ab}}$ & $1.18 \pm 0.02^{b}$ \\
\hline
\end{tabular}

Values are mean \pm SEM $(n=6)$

Values with different superscript letters are significantly different $(P<0.05)$

characterized by retention of biliary pigment usually found in the portal-space area and may reach as far as the lobe periphery (Fig. 2c). Liver of ANIT + TvE rats showed mild hydropic degeneration of hepatocytes and mild leukocytes infiltration (Fig. 2d). Liver of ANIT + UDCA rats showed normal hepatocytes, mild congestion of central vein and weak bleeding (Fig. 2e). Liver of ANIT + TvE + UDCA rats showed a normal architecture, with hepatic lobules around the central vein and each lobule consisting of hepatic cords of hepatocytes (Fig. 2f). Masson's trichrome stain localized high distribution of the collagen fibers in the portal-space area in the ANIT group as compared to other groups (Fig. 3).

\section{Immunohistochemistry}

Immunohistochemical staining of caspase-3 was localized in the nuclei and cytoplasm of hepatocytes. The liver tissues of the control and TvE groups showed weak expression of caspase-3 (Figs. 4a, b and 6a), whereas significant strong expression was observed in the ANIT group as compared to control group (Fig. 4a, c). Liver of ANIT + TvE rats showed significant weak expression of caspase-3 as compared to ANIT group (Figs. 4d and 6a). Liver of ANIT + UDCA rats showed significant weak expression of caspase- 3 as compared to ANIT group (Figs. 4e and 6a). Liver of ANIT + TvE + UDCA rats showed significant weak expression of caspase- 3 as compared to ANIT group (Figs. 4f and 6a).

Immunohistochemical staining of $\mathrm{Bcl}-2$ was localized in the nuclei and cytoplasm of hepatocytes. The liver tissues of the control group showed moderate expression of Bcl-2 proteins (Figs. 5a and $6 \mathrm{~b}$ ). The liver tissues of the $\mathrm{TvE}$ group showed strong expression of Bcl-2 as compared to ANIT group (Figs. 5b and 6b), whereas weak expression was observed in the ANIT group as compared to control group (Figs. 5c and 6b). Liver of ANIT + TvE rats showed a significant increase in the expression of $\mathrm{Bcl}-2$ protein as compared to ANIT group (Figs. 5d and 6b). Liver of ANIT + UDCA rats showed strong expression of $\mathrm{Bcl}-2$ (Figs. $5 \mathrm{e}$ and $6 \mathrm{~b}$ ). Liver of ANIT + TvE + UDCA rats showed nonsignificant weak expression as compared to ANIT group (Figs. $5 \mathrm{f}$ and $6 \mathrm{~b}$ ).

\section{Discussion}

Chronic liver disease represents a major health problem worldwide. The progress of chronic liver disease into hepatic fibrosis, cirrhosis, and/or hepatocellular carcinoma has become an important cause of morbidity and mortality (Rajapaksha, Angus, \& Herath, 2019). According to the finding of Mohajeri et al. (2019), the PSC and $\mathrm{PBC}$ are the most important causes of morbidity and mortality for patients with liver disease that presumed to be autoimmune diseases. The current available therapy for cholestatic and autoimmune hepatitis $(A I H)$ is UDCA and obeticholic acid (OCA) (Hirschfield et al., 2015). However, about 25 to $40 \%$ of patients with PBC did not achieve a complete treatment response (Chascsa, Carey, \& Lindor, 2017). Therefore, there is an urgent demand to develop new therapies beyond UDCA and OCA that are aimed at both slowing disease course and improving quality of life. In addition, Liberal et al. (2017) reported that stimulation and perpetuation of $\mathrm{AIH}$ mainly occurred due to imbalance between Treg and effector $\mathrm{T}$ cells. Therefore, it is important to develop a new drug to modulate both Th1 and Th2 cells balance. Thereby, the ongoing study was proved the ability of TvE to modulate the rat immune response during experimental cholangitis induced by ANIT.

In the assessment of liver toxicity by ANIT, the determination of enzyme levels, such as serum AST, ALT, and GGT considered as a golden standard assessment for accurate detection and early diagnosis of liver injury. It has been shown that the obstruction of bile drainage 


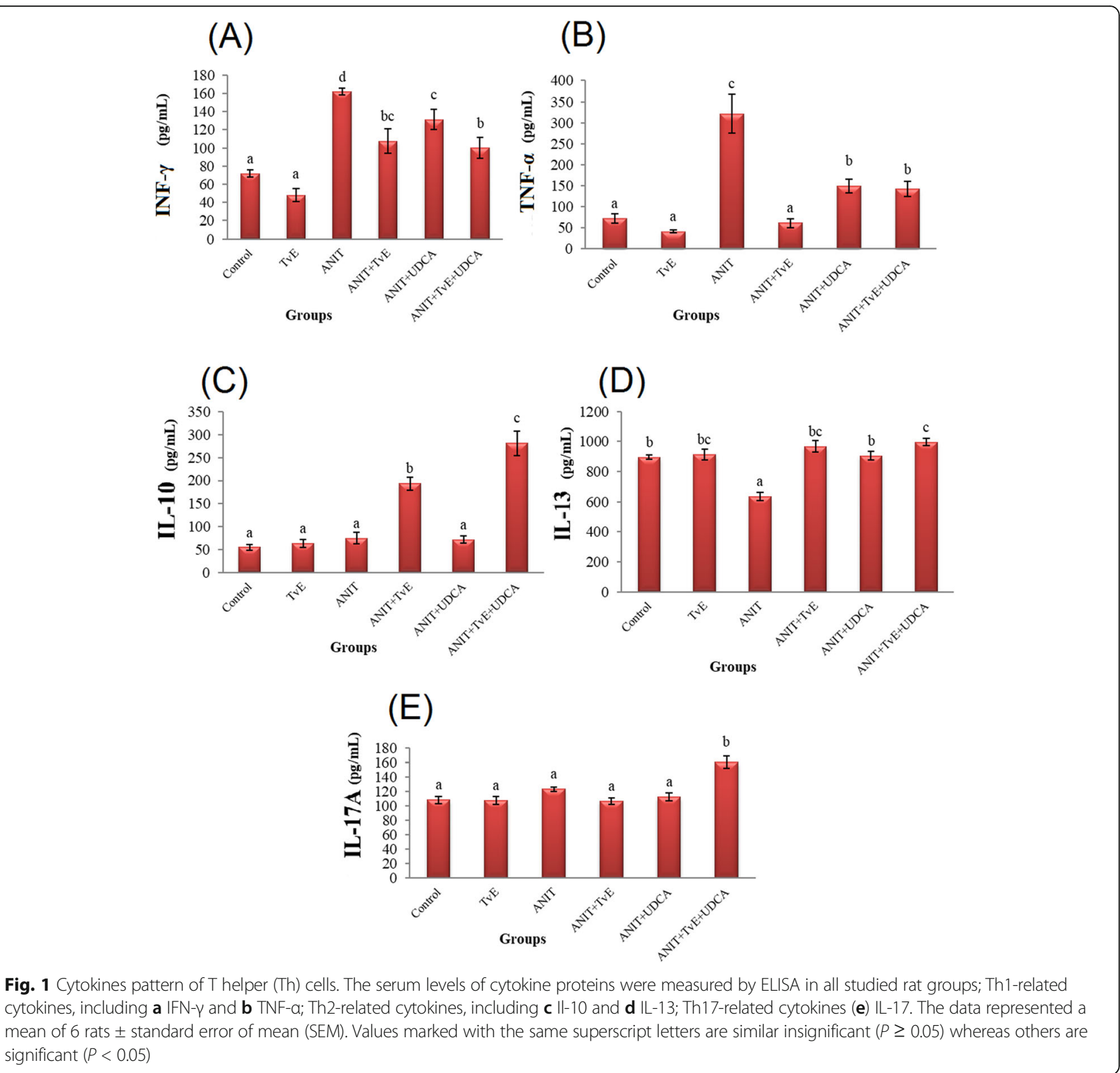

in the intestine, with the consequent retention of bile constituents in the liver is responsible for the liver function disorder (Damnjanović et al., 2013). Bile acids collect in hepatocytes a mild cholestasis and have the potential to cause cytotoxicity (Nassan, Ismail, Soliman, \& Alkhedaide, 2018). In the present study, the underlying mechanism of normalization in the levels of the studied enzymes in the animals treated firstly with TvE or UDCA may indicate maintenance of functional integrity of hepatic cell membrane and restoration of their architecture. Histopathological and immunohistological findings in the current study affirmed the study of Dilger et al. (2012) showing that cholestasis leads to apoptosis and inflammation in hepatocytes and loss of normal architecture of the liver.

In the condition of hepatic diseases, serum bilirubin is one of the most sensitive biomarkers. In accord with the report of Chen et al. (2016), our investigation showed that administration of ANIT caused a marked increase in total bilirubin level. However, treatment with TvE prior to ANIT administration causes a distinct decrease in total bilirubin as compared to ANIT group. The current investigation recorded important microscopic histological alterations which indicate biliary pigment retention within the hepatic lobes. Necrosis may follow virtually any lesion whose changes are significant, taking 


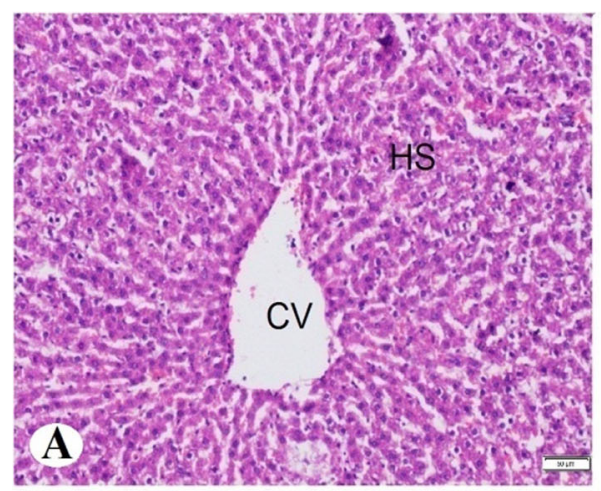

\section{Control}

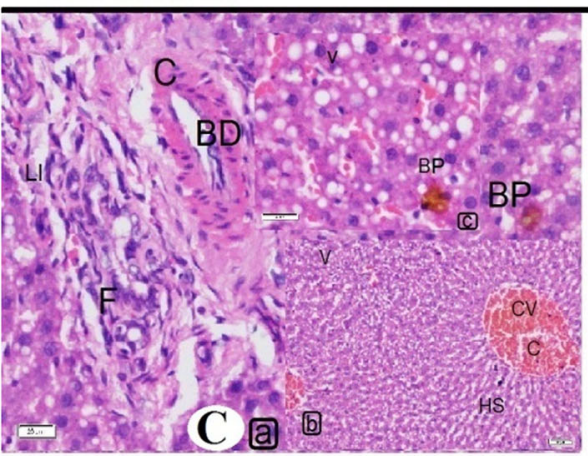

ANIT

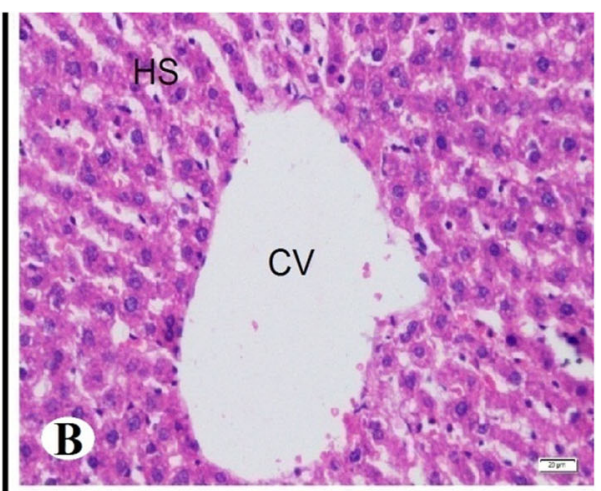

TvE

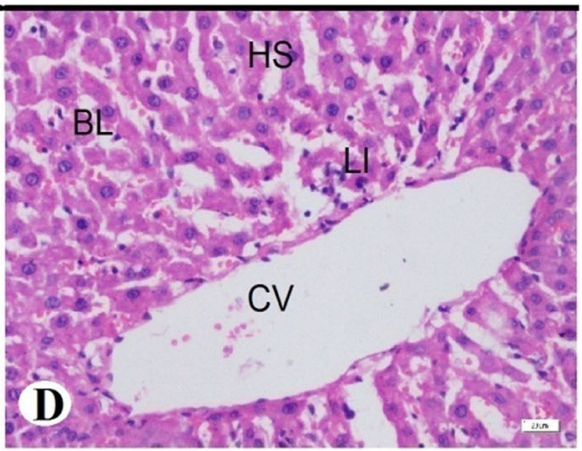

ANIT + TvE

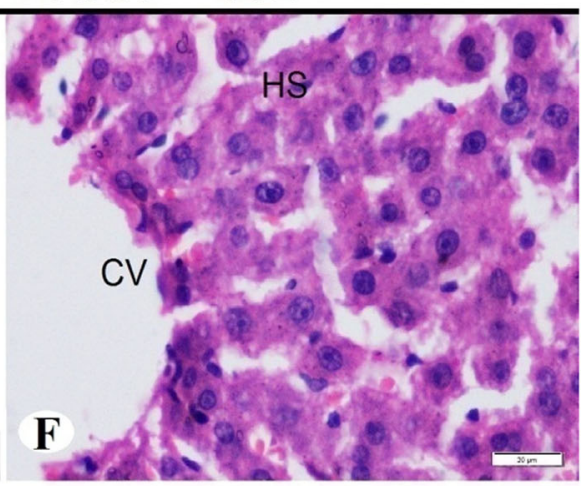

ANIT + TvE + UDCA

\section{ANIT + UDCA}

\section{Stain : H \& E}

Fig. 2 Photomicrographs of liver tissues in all rat groups stained by hematoxylin and eosin (H\&E staining). a Control group showing the normal architecture of the liver with hepatic lobules around the central vein (CV) and each lobule consisting of hepatic strands (HS) of hepatocytes (scale bar $=50 \mu \mathrm{m})$. $\mathbf{b}$ (TvE) group showing normal hepatic strands (HS) of hepatocytes around the central vein (CV) (scale bar $=20 \mu \mathrm{m})$. c (C.a) ANIT group showing leukocytes infiltration (L)), fibrosis (F), bilirubin pigment (BP), and congestion (C) in the bile duct (BD). (C.b) showing vaculated cells $M$ ) and severe congestion (C) in central vein (CV). (C.C) showing bilirubin pigment (BP) (scale bar $a=20 \mu \mathrm{m}, b=50 \mu \mathrm{m}, \mathrm{c}=20 \mu \mathrm{m}$ ). $\mathbf{d}$ ANIT + TVE group showing mild hydropic degeneration of hepatocytes around the central vein (CV), bleeding (BL), and leukocytic in filtration (LI) (scale bar $=20 \mu \mathrm{m})$. e ANIT + UDCA group showing normal hepatocytes and mild congestion of central vein (cv) (scale bar $=20 \mu \mathrm{m})$. f ANIT + AIE + UDCA group showing the normal architecture of the liver, with hepatic lobules around the central vein (CV) and each lobule consisting of hepatic strands (HS) of hepatocytes (scale bar $20 \mu \mathrm{m}$ )

a toll on hepatocytes. Retained biliary material may have a swollen, frothy, and diffuse aspect.

Oxidative stress is a systemic phenomenon in regulating cellular protein post-translational modifications
(Assimakopoulos et al., 2006). It was reported that the formation of reactive oxygen species (ROS) during chronic inflammation induced liver injury (Bessa, Mohamed, Abd El-Wahab, \& Nor El-Din, 2012). Due to 


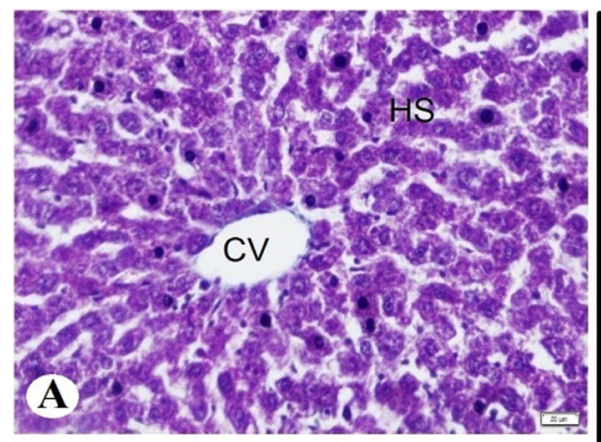

\section{Control}

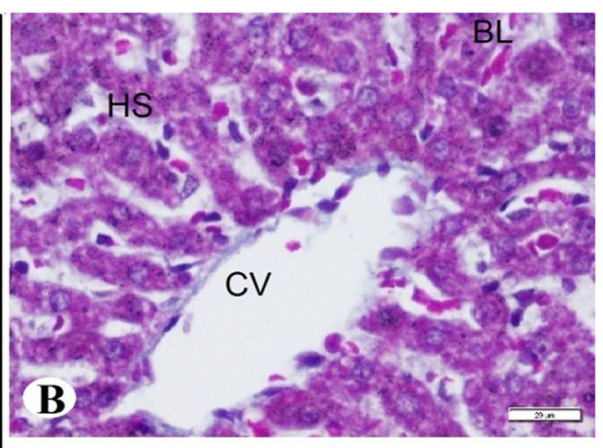

TvE
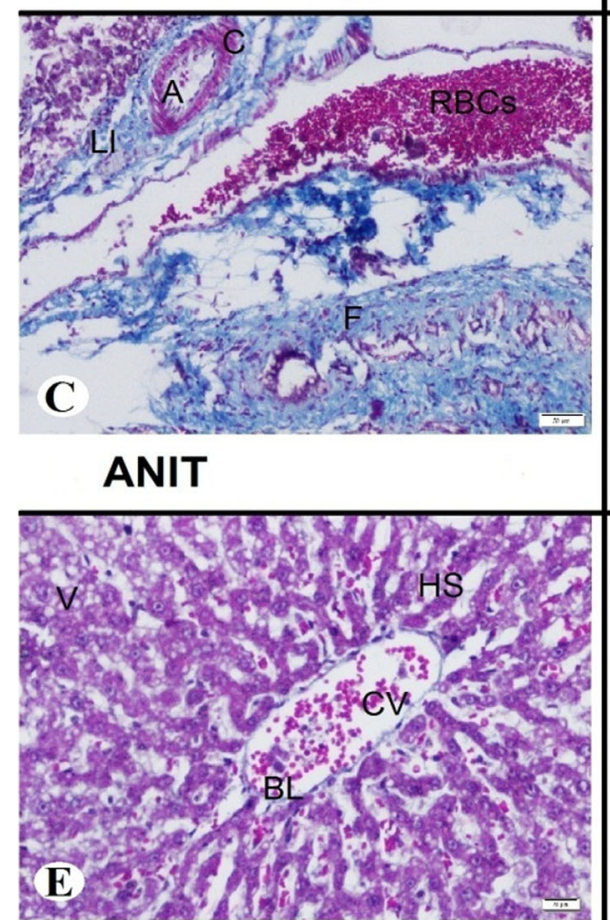

ANIT + UDCA

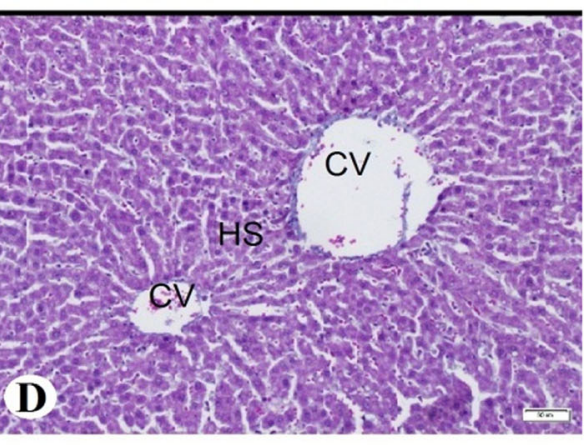

ANIT + TVE

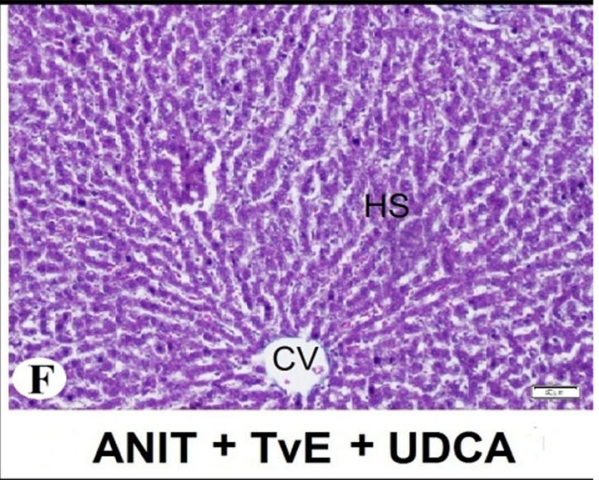

\section{Stain : Masson's trichrome stain}

Fig. 3 Photomicrographs of liver tissues for fibrosis evaluation in all rat groups, stained with Masson's trichrome stain. a Control group showing the normal architecture of the liver, with hepatic strands (HS) around the central vein (CV) (scale bar $20 \mu \mathrm{m}$ ). b (TvE) group showing the normal architecture of the liver, with hepatic strands (HS) around the central vein (CV) (scale bar $20 \mu \mathrm{m})$. c ANIT group showing severe fibrosis (F) around hepatic vein and hepatic artery (A), congestion (C) in hepatic vein and leukocytes infiltration (LI) (scale bar $=50 \mu \mathrm{m}$ ). $\mathbf{d}$ ANIT + TvE group showing the normal architecture of the liver, with hepatic strands $(\mathrm{HS})$ around the central vein $(\mathrm{CV})($ scale bar $=50 \mu \mathrm{m})$. e ANIT + UDCA group showing normal hepatic strands (HS) around the central vein (CV) and mild bleeding (scale bar $=20 \mu \mathrm{m}$ ). $\mathbf{f}$ ANIT + AIE + UDCA group showing the normal architecture of the liver, with hepatic lobules around the central vein (CV) and each lobule consisting of hepatic strands (HS) of hepatocytes (scale bar $50 \mu \mathrm{m}$ )

the enhancement in the levels of lipid peroxidation end product, MDA can be used as an indicator of the intensity of oxidative stress (Damnjanović et al., 2013). The present investigation showed a significant increment in the level of MDA following ANIT administration that may suggest enhanced lipid peroxidation leading to tissue damage and failure of antioxidant defense mechanisms to prevent formation of excessive free radicals
(Tsuchiya, Kirima, Yoshizumi, \& Tamaki, 2003). The data were confirmed by immunohistochemical finding, where expression levels of $\mathrm{Bcl}-2$ proteins were decreased, and the levels of caspase- 3 proteins increased to enhance apoptosis of hepatocytes in ANIT group. Accumulating event suggests that free radicals are associated with apoptosis and lipid peroxidation in cholestatic lesions (Yang, Ramani, \& Xia, 2009). However, the decrease of 

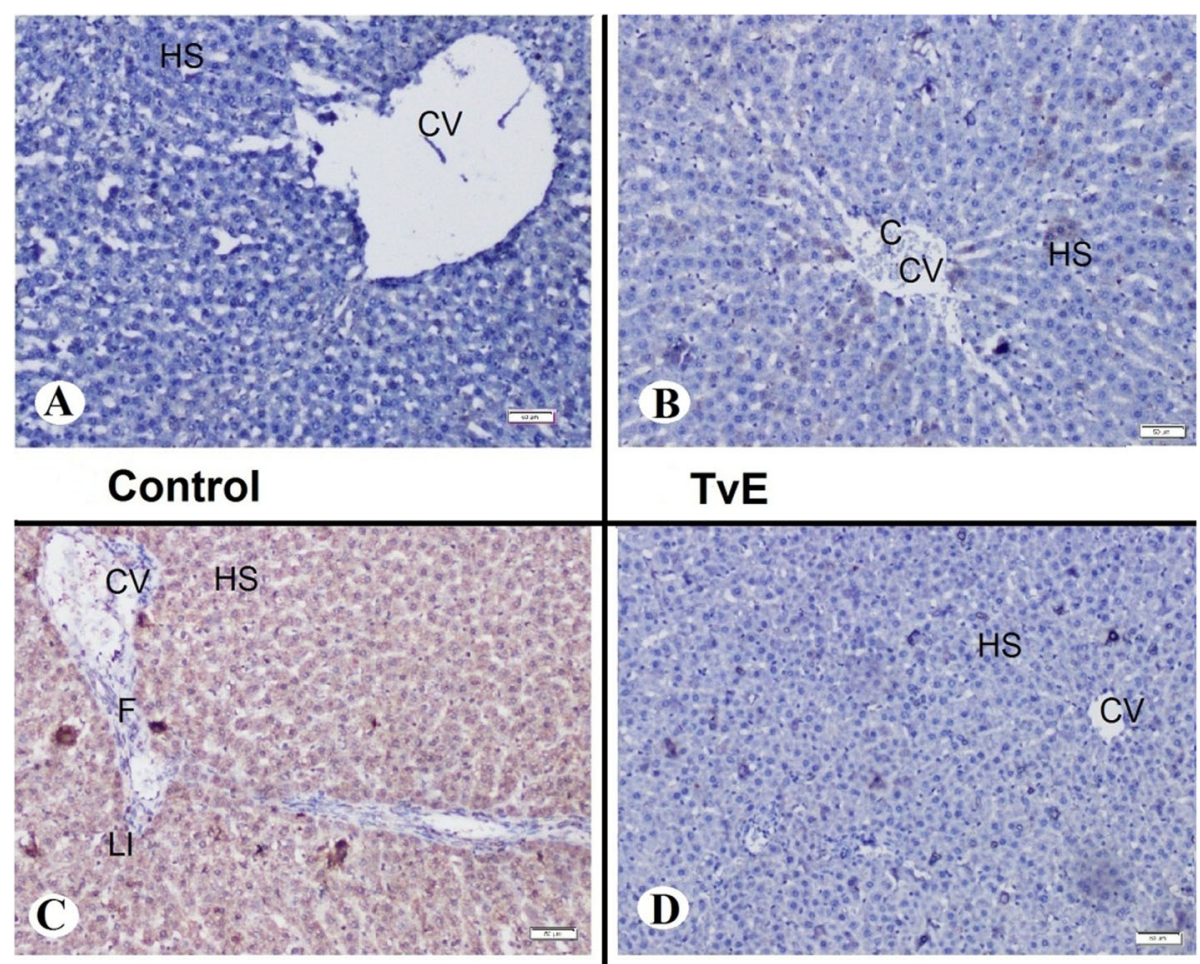

\section{TvE}
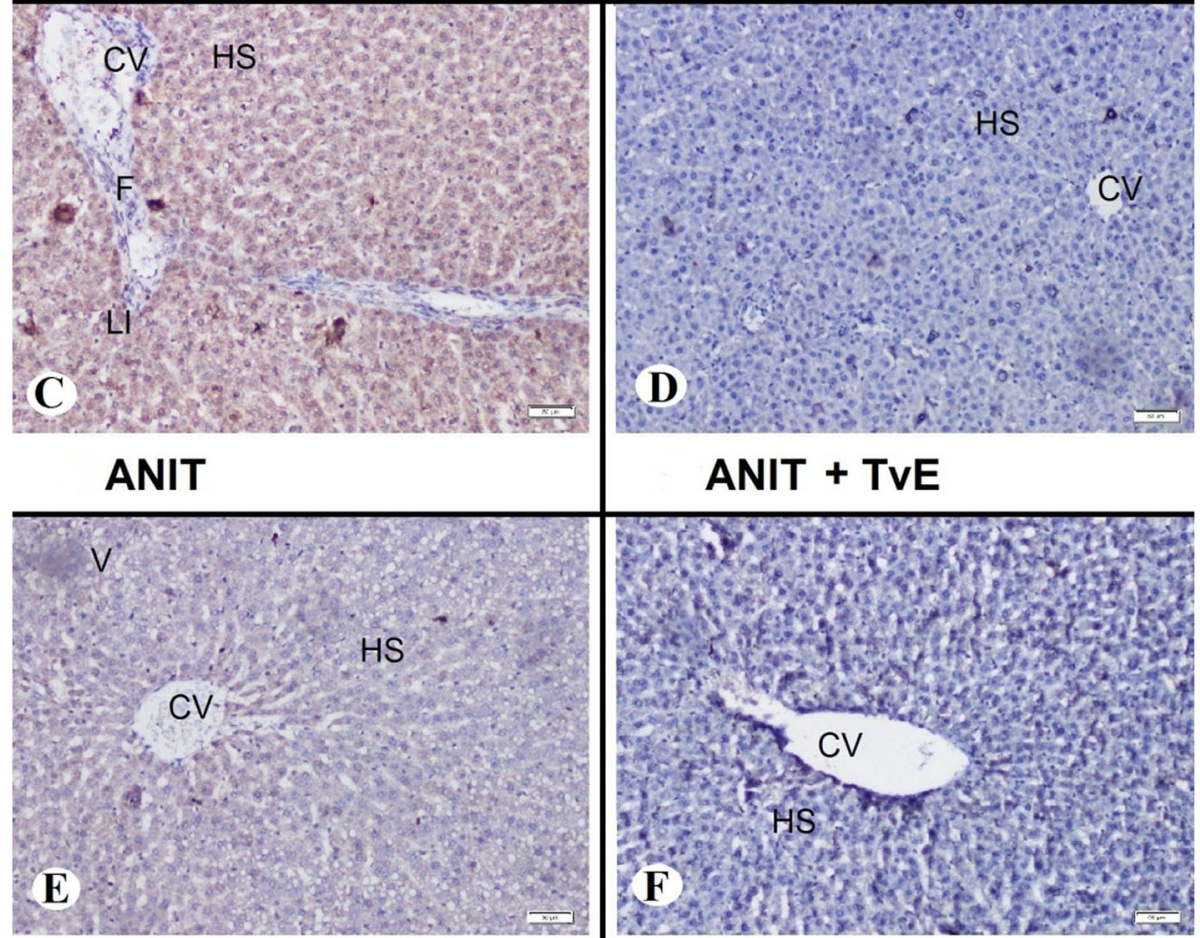

ANIT + UDCA

ANIT + TvE

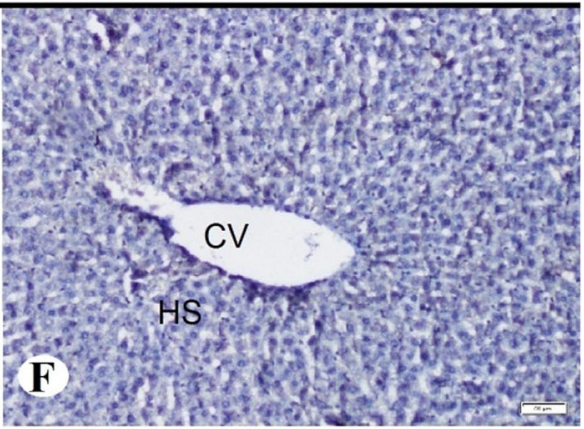

ANIT + TvE + UDCA

\section{Stain : Caspase-III}

Fig. 4 Photomicrographs of hepatocytes of rats showing cellular distribution of immunoreactive caspase-3 protein as indicated by brown color. a Control group showing weak reaction. b (TVE) group showing weak reaction. c ANIT group showing strong positive reaction. $\mathbf{d}$ ANIT + TVE group showing weak reaction. e ANIT+UDCA group showing weak reaction. f ANIT + TVE + UDCA group showing weak reaction of caspase-3. Scale bar $=50 \mu \mathrm{m}$

lipid peroxidation-mediated oxidative stress may be a potential and effective strategy for the prevention and treatment of hepatic failure (Tsuchiya et al., 2003). Indeed, application of $\mathrm{TvE}$ ( $\mathrm{TvE}$ group) in the present study did not directly affect the total GSH and the antioxidant enzyme, CAT.

IFN- $\gamma$ is one of the predominant cytokines secreted by activated Th1 and cytotoxic T cells. The present study showed an increase in the systemic serum levels of IFN$\gamma$ and TNF- $\alpha$ which indicated the differentiation towards
Th1 immune response in the acute cholangitis rat model compared to control rats. Neutrophils have been recruited to the liver under the influence of proinflammatory mediators that provoked by accumulated bile acids (Cai et al., 2017). Moreover, Tjandra, Sharkey, and Swain (2000) recorded an increase in the hepatic mRNA expression of IL-12, the cytokine derivedmacrophage-activated Th1. It was reported that IFN- $\gamma$ was described to induce hepatocellular apoptosis and DNA fragmentation and leakage (Morita, Watanabe, \& 


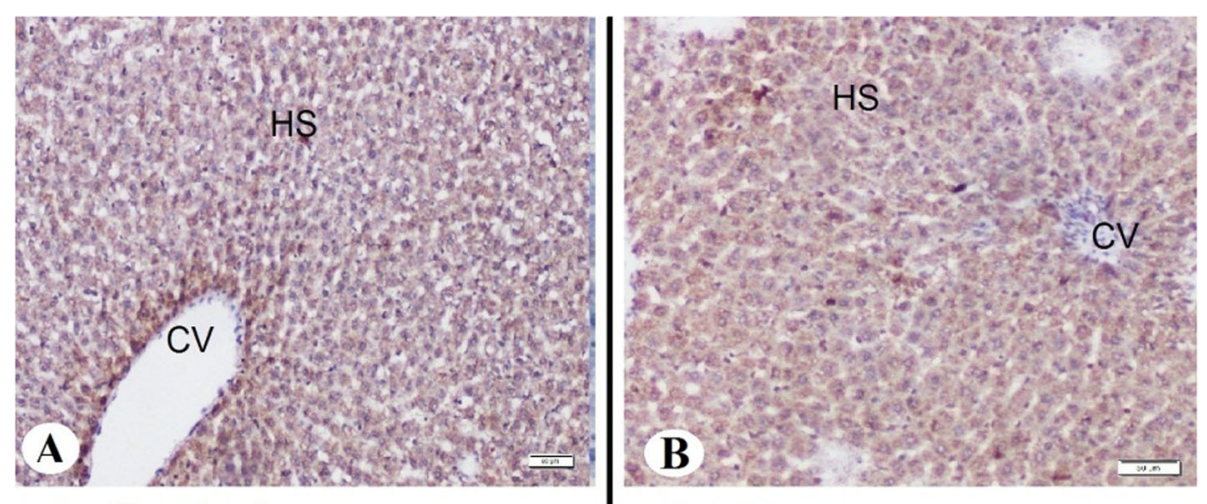

TvE
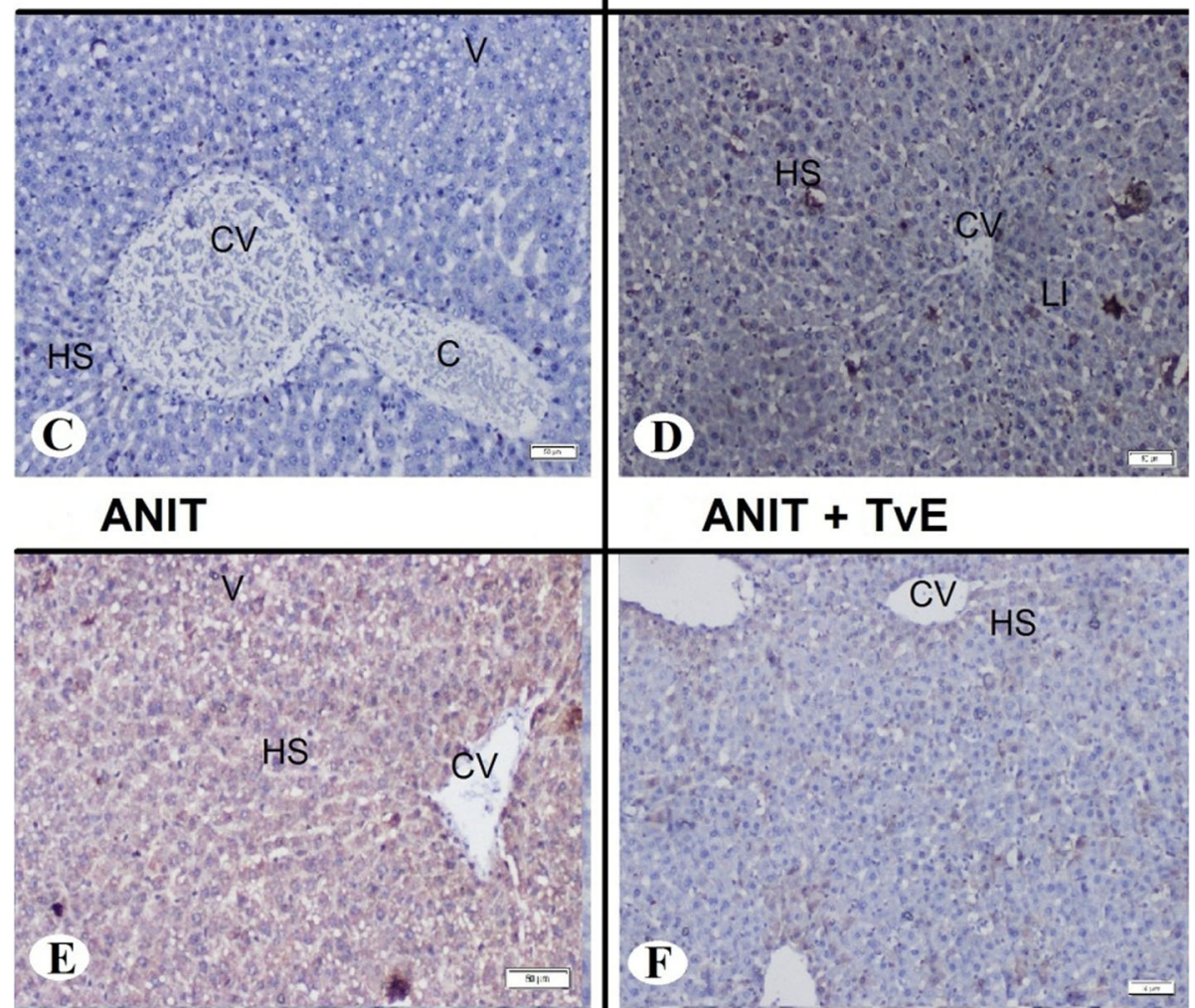

ANIT + TvE
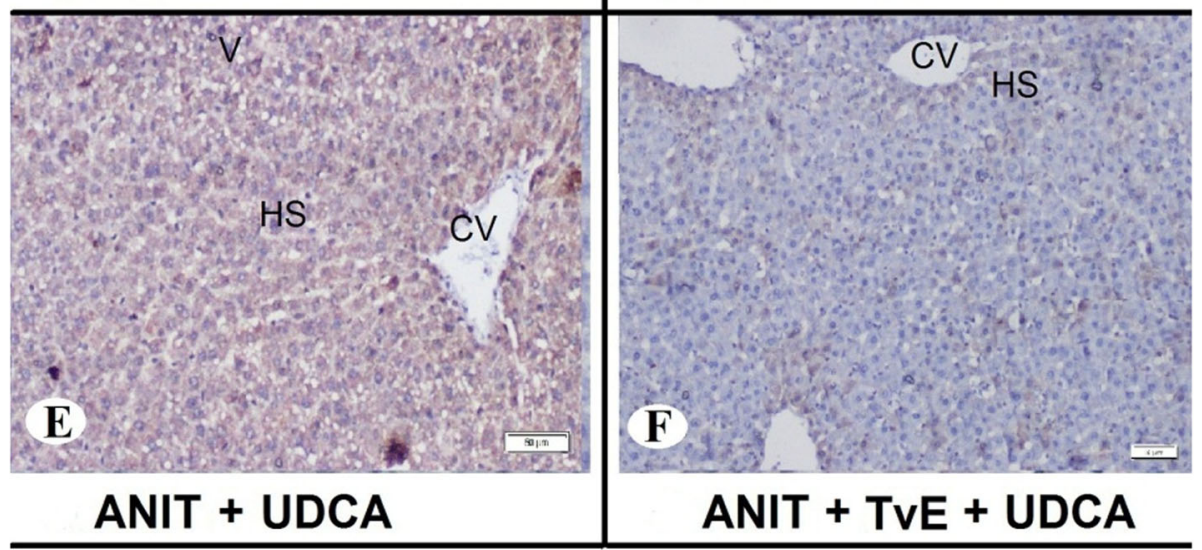

Stain : BCl -II

Fig. $5 \mathrm{Imm}$ unohistochemical staining of liver with $\mathrm{BCl}$ 2. Photomicrographs of hepatocytes of rats showing cellular distribution of immunoreactive Bcl2 protein as indicated by brown color. a Control group showing strong positive reaction. b TVE group showing strong positive reaction. $\mathbf{c}$ ANIT group showing weak reaction. $\mathbf{d}$ ANIT + TVE group showing strong positive reaction. e ANIT + UDCA group showing strong positive reaction. $\mathbf{f}$ ANIT + TVE + UDCA group showing weak reaction. Scale bar $=50 \mu \mathrm{m}$

Akaike, 1995). The present study also showed that the increase in the expression of caspase- 3 and decrease Bcl2 are in conjunction with the IFN- $\gamma$ elevation.

In addition, ANIT-treated rats in the present study showed a significant decrease in the IL-13 (Th2-related cytokines). Thereby, the present investigation revealed converse relation between the IFN- $\gamma$ production and suppression of IL-13. There is a growing evidence that liver cells are populated with other plastic phenotypes of macrophages that may be stimulated by IFN- $\gamma$ and subsequently can secrete proinflammatory cytokines such as TNF- $\alpha$, IL- $1 \beta$, and ROS, which participate in liver inflammation and disease progression (Tacke, 2017).

IL-17A is one of the major cytokines secreted by Th17, a key player to recruit and activate neutrophils and monocytes as well as targeting nonimmune cells to provoke 

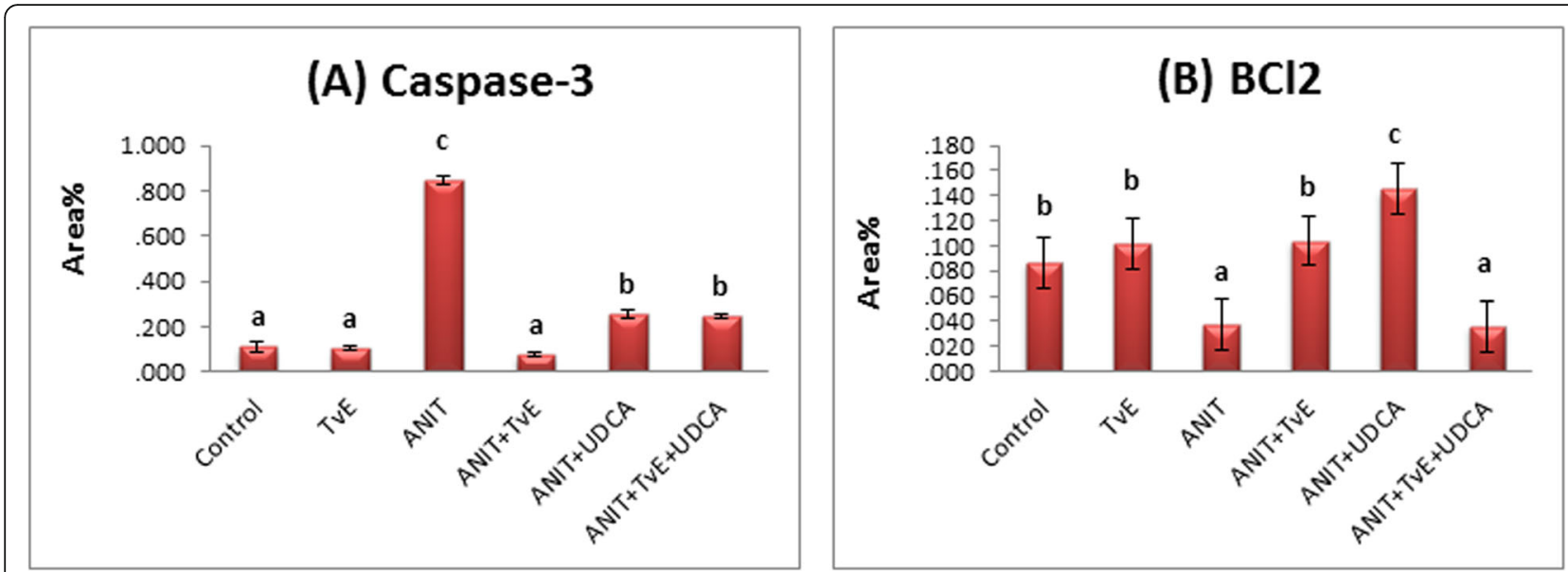

Fig. 6 Histogram of area \% of (a) caspase-3 and (b) Bcl-2 protein in liver cells. Expression positivity of caspase-3 and Bcl-2 in hepatocytes of rats was presented as mean \pm standard error of the mean (SEM). Values with different superscript letters are significantly different $(P<0.05)$

mediators of inflammation (Korn, Bettelli, Oukka, \& Kuchroo, 2009). In the present study, ANIT-treated rats displayed a slight increase in the levels of IL-17 compared to control rats. In contrast, various research studies have evidenced the relation between PBC and Th17 in humans and animals (Fenoglio et al., 2012).

Protection and pathological responses against pathogens depend on the balance of Th1 and Th2 immune responses (Abbas, Murphy, \& Sher, 1996). In different types of nematodes, IFN- $\gamma$ plays a crucial role in stimulation of protective immunity or chronic disease persistence. In the current work, treatment with $\mathrm{TVE}$ showed lower levels of IFN- $\gamma$ compared to ANIT-rats. Also, mice immunized with somatic antigen of $T$. canis decreased production of IFN- $\gamma$ (Dvorožňáková, Borošková, \& Tomašovičová, 2002). The soluble products of intestinal helminths have been found to suppress both Th1 and Th17, which are central participants in the immunopathogenesis of autoimmune disorders (Kuijk et al., 2012). The current study showed that TvE increased the levels of $\mathrm{Bcl}-2$ protein and decreased the levels of caspase- 3 protein, decreasing apoptosis, suggesting that suppression of apoptosis may be one potential mechanism of TvE against ANIT-induced liver cholangitis in rats.

IL-10 is known as anti-inflammatory cytokine and produced by activated regulatory $\mathrm{T}$ cells down regulating the inflammatory immune responses (Sakaguchi, 2004). Increase levels of Th2 cytokines (IL-4, IL-5, and IL-13) as a result of interaction between helminth and epithelial cells during helminth infections polarized CD4 T cells to Th2 phenotype (Maizels \& McSorley, 2016). Toxocariasis infection is depicted in triggering macrophages to secrete IL-4 that promotes Th2-responses by increment the levels of IL-5, IL-6, and IL-13 (Allen \& Maizels, 2011) The current work showed that TvE can immunomodulate the ANIT-specific cholangitis by a marked decrease in Th1 cytokines (IFN- $\gamma$ and TNF- $\alpha$ ) and an increase in Th2 (IL-10 and IL-13) production. Our results support the protective adaptive mechanisms as elevated IL-10 levels are accompanied with suppression of proinflammatory cytokines (TNF- $\alpha$ and IFN- $\gamma$ ). It is known that UDCA is the anti-inflammatory drug used in the treatment of liver disease (Joo, Kang, Won, \& Lee, 2003). It can suppress the proinflammatory cytokines (TNF- $\alpha$, IL-1 $\alpha, \beta$, and IL-6) (Ko et al., 2017), and increases the expression of anti-inflammatory cytokine IL-10 for $24 \mathrm{~h}$ (Clarke, Hales, Hunt, \& Foxwell, 1998). On the same line, $\mathrm{TvE}$ alone showed a marked decrease in the inflammatory cytokines (IFN- $\gamma$ and TNF- $\alpha$ ) synergize with the increase of anti-inflammatory cytokines (IL-10 and IL13) in cholangitis-induced rats as compared to rats treated with UDCA. These results confirmed the proposed immunomodulatory effect of $\mathrm{TvE}$ on cholangitis.

\section{Conclusions}

The obtained results give a sound on the proposed mechanistic efficacy of $\mathrm{TvE}$ as a protective strategy in liver cholangitis. TvE demonstrated profound immunomodulatory impact on the polarization state from the dominant Th1 to Th2. The upregulation of IL-10 and IL-13 is synergized with downregulation of IFN- $\gamma$ and TNF- $\alpha$. TvE could be suggested as a potential therapeutic alternative in cholestasis. TvE increased the levels of $\mathrm{Bcl}-2$ protein and decreased the levels of caspase-3 protein to reduce apoptosis, suggesting that suppression of apoptosis may be one potential mechanism of $\mathrm{TvE}$ against ANIT-induced liver cholangitis in rats.

\section{Abbreviations}

TVE: Toxocara vitulorum extract; ANIT: Alpha-naphthylisothiocyanate; UDCA: Ursodeoxycholic acid; AST: Aspartate aminotransferase; ALT: Alanine aminotransferase; ALP: Alkaline phosphatase; GGT: Glutamyltransferase; 
MDA: Malondialdehyde; GSH: Glutathione reduced; CAT: Catalase; Th: T helper; IFN: Interferon- $\gamma_{i}$ TNF: Tumor necrosis factor-a; IL: Interleukin

\section{Acknowledgements}

All authors would to thank Cairo University for equipment's support.

\section{Authors' contributions}

AMB contributed to cytokine assays, data analysis, and interpretation of data. MF contributed to all practical work, acquisition, and statistical analysis. AAB contributed to histological examination, immunohistochemical analysis, and interpretation of data. ASM contributed to measuring of liver functions and oxidative stress markers, and interpretation of data. NAM contributed to cytokine assays, data analysis, and interpretation of data. SRF is mainly responsible for study design and planning, acquisition, and supervision. The manuscript is read, drafted, and approved by all authors.

\section{Funding}

The author(s) received no financial support for the research.

\section{Availability of data and materials}

All data and materials presented in the manuscript are original work of the authors. The authors can be contacted for any additional supporting data required by the journal.

\section{Ethics approval and consent to participate}

Experimental protocols and procedures used in this study were approved by the Cairo University-Institutional Animal Care and Use Committee (CU-IACUC) (Egypt), (approval no. CU/I/F/95/17).

\section{Consent for publication}

Not applicable.

\section{Competing interests}

All authors declare that there is no conflict of interest.

Received: 30 August 2020 Accepted: 25 November 2020

Published online: 11 December 2020

\section{References}

Abbas, A. K., Murphy, K. M., \& Sher, A. (1996). Functional diversity of helper T lymphocytes. Nature, 383, 787-793.

Abe, M., Hiasa, Y., \& Onji, M. (2013). T helper 17 cells in autoimmune liver diseases. Clinical and Developmental Immunology, 2013, 607073

Alkhedaide, A. Q., Ismail, T. A., Alotaibi, S. H., Nassan, M. A., \& Shehri, Z. S. (2018) Preventive effect of artemisinin extract against cholestasis induced via lithocholic acid exposure. Bioscience Reports, 38(6), BSR20181011.

Allen, J. E., \& Maizels, R. M. (2011). Diversity and dialogue in immunity to helminths. Nature Reviews Immunology, 11, 375-388.

Assimakopoulos, S., Thomopoulos, K., Patsoukis, N., Georgiou, C., Scopa, C., Nikolopoulou, V., et al. (2006). Evidence for intestinal oxidative stress in patients with obstructive jaundice. European Journal of Clinical Investigation, 36(3), 181-187.

Bessa, S., Mohamed, A. E., Abd El-Wahab, A.-S., \& Nor El-Din, S. (2012). Heme oxygenase-1 mRNA expression in egyptian patients with chronic liver disease. Hepatitis Monthly, 12(4), 278-285.

Cai, S., Ouyang, X., Chen, Y., Soroka, C., Wang, J., Mennone, A., et al. (2017). Bile acids initiate cholestatic liver injury by triggering a hepatocyte-specific inflammatory response. JCl Insight, 9.2(5), 90780

Caraballo, L., \& Acevedo, N. (2011). New allergens of relevance in tropical regions: The impact of Ascaris lumbricoides infections. World Allergy Organization Journal, 4(5), 77-84

Chascsa, D., Carey, E., \& Lindor, K. (2017). Old and new treatments for primary biliary cholangitis. Liver International, 37(4), 490-499.

Chen, H., Huang, X., Min, J., Li, W., Zhang, R., Zhao, W., et al. (2016). Geniposidic acid protected against ANIT-induced hepatotoxity and acute intrahepatic cholestasis, due to Fxr-mediated regulation of Bsep and Mrp2. Journal of Ethnopharmacology, 179, 197-207.

Clarke, C., Hales, A., Hunt, A., \& Foxwell, B. (1998). IL-10-mediated suppression of TNF-a production is independent of its ability to inhibit NFkB activity. European Journal of Immunology, 28(5), 1719-1726.
Crispe, I. (2009). The liver as a lymphoid organ. Annual Review of Immunology, 27, 147-163.

Damnjanović, Z., Jovanović, M., Nagorni, A., Radojković, M., Sokolović, D., Damnjanović, G., et al. (2013). Correlation of inflammation parameters and biochemical markers of cholestasis with the intensity of lipid peroxidation in patients with choledocholithiasis. Vojnosanitetski Pregled, 70(2), 170-176.

Dilger, K., Hohenester, S., Winkler-Budenhofer, U., Bastiaansen, B., Schaap, F., Rust, C., et al. (2012). Effect ofursodeoxycholic acid on bile acid profiles and intestinal detoxification machinery in primary biliarycirrhosis and health. Journal of Hepatology, 57, 133-140.

Dvorožňáková, E., Borošková, O., \& Tomašovičová, O. (2002). Immune responses in mice immunized with Toxocara canis antigens. Helminthologia, 39(2), 59-66.

Erb, K. J. (2007). Helminths, allergic disorders and IgE-mediated immune responses: Where do we stand? European Journal of Immunology, 37, 1170-1173.

Fenoglio, D., Bernuzzi, F., Battaglia, F., Parodi, A., Kalli, F., Negrini, S., et al. (2012). Th17 and regulatory $T$ lymphocytes in primary biliary cirrhosis and systemic sclerosis as models of autoimmune fibrotic diseases. Autoimmunity Reviews, 12(2), 300-304

Fouser, L., Wright, J., Dunussi-Joannopoulos, K., \& Collins, M. (2008). Th17 cytokines and their emerging roles in inflammation and autoimmunity. Immunological Reviews, 226, 87-102.

Goddey, N., Osagie, I., \& Maliki, A. (2010). Serum cytokines profiles in Nigerian children with Ascaris infection. Asian Pacific Journal of Tropical Medicine, 3(4), 288-291.

He, H., Mennone, A., Boyer, J., \& Cai, S. (2011). Combination of retinoic acid and ursodeoxycholic acid attenuates liver injury in bile duct-ligated rats and human hepatic cells. Hepatology, 53(2), 548-557.

Heymann, F., \& Tacke, F. (2013). Immunology in the liver--from homeostasis to disease. Nature Reviews Gastroenterology \& Hepatology, 13(2), 88-110.

Hirschfield, G., Mason, A., Luketic, V., Lindor, K., Gordon, S., Mayo, M., et al. (2015). Efficacy of obeticholic acid in patients with primary biliary cirrhosis and inadequate response to ursodeoxycholic acid. Gastroenterology, 148(4), 751-761.

Hua, H., Dai, M., Luo, Y., Lin, H., Xu, G., Hu, X., et al. (2019). Basal PPARa inhibits bile acid metabolism adaptation in chronic cholestatic model induced by anaphthylisothiocyanate. Toxicology Letters, 300, 31-39.

Jin, F., Cheng, D., Tao, J.-Y., Zhang, S.-L., Pang, R., Guo, Y.-J., et al. (2013). Antiinflammatory and anti-oxidative effects of corilagin in a rat model of acute cholestasis. BMC Gastroenterology, 13, 79

Joo, S., Kang, H., Won, T., \& Lee, D. (2003). Ursodeoxycholic acid inhibits proinflammatory repertoires, IL-1 beta and nitric oxide in rat microglia. Archives of Pharmacal Research, 26(12), 1067-1073.

Ko, W.-K., Lee, S.-H., Kim, S., Jo, M.-J., Kumar, H., Han, I.-B., et al. (2017). Antiinflammatory effects of ursodeoxycholic acid by lipopolysaccharide-stimulated inflammatory responses in RAW 264.7 macrophages. PLoS One, 12(6), e0180673.

Kodali, P., Wu, P., Lahiji, P., Brown, E., \& Maher, J. (2006). ANIT toxicity toward mouse hepatocytes in vivo is mediated primarily by neutrophils via CD18. American Journal of Physiology-Gastrointestinal and Liver Physiology, 291(2), 355-363.

Korn, T., Bettelli, E., Oukka, M., \& Kuchroo, V. (2009). IL-17 and Th17 Cells. Annual Review of Immunology, 27, 485-517.

Kuijk, L. M., Klaver, E. G., Kooij, G., van der Pol, S. M., Heijnen, P., et al. (2012). Soluble helminth products suppress clinical signs in murine experimental autoimmune encephalomyelitis and differentially modulate human dendritic cell activation. Molecular Immunology, 51, 210-218.

Li, Y., Yu, H., Xu, Z., Shi, S., Wang, D., Shi, X., et al. (2019). Melatonin ameliorates ANIT induced cholestasis by activating Nrf2 through a PI3K/Akt dependent pathway in rats. Molecular Medicine Reports, 19(2), 1185-1193.

Liberal, R., de Boer, Y. S., Andrade, R., Bouma, G., Dalekos, G., Floreani, A., et al. (2017). Expert clinical management of autoimmune hepatitis in the real world. Alimentary Pharmacology \& Therapeutics, 45(5), 723-732.

Lleo, A., Marzorati, S., Anaya, J., \& Gershwin, M. E. (2017). Primary biliary cholangitis: A comprehensive overview. Hepatology International, 11(6), 485-499.

Maizels, R., \& McSorley, H. (2016). Regulation of the host immune system by helminth parasites. Journal of Allergy and Clinical Immunology, 138, 666-675.

Mohajeri, S., Bezabeh, T., ljare, O., King, S., Thomas, M., Minuk, G., et al. (2019). In vivo $1 \mathrm{H}$ MRS of human gallbladder bile in understanding the pathophysiology of primary sclerosing cholangitis (PSC): Immune-mediated disease versus bile acid-induced injury. NMR in Biomedicine, 32(5), 4065.

Morita, M., Watanabe, Y., \& Akaike, T. (1995). Protective effect of hepatocyte growth factor on interferon-gamma-induced cytotoxicity in mouse hepatocytes. Hepatology, 21, 1585-1593. 
Nassan, M., Ismail, T., Soliman, M., \& Alkhedaide, A. (2018). Ameliorative effect of curcumin and vitamin B6 against lithocholic acid-induced cholestasis and liver injury in mice. Brazilian Journal of Veterinary Pathology, 11, 50-63.

Ohta, Y., Kongo, M., Sasaki, E., \& Harada, N. (1999). Change in hepatic antioxidant defense system with liver injury development in rats with a single alphanaphthylisothiocyanate intoxication. Toxicology, 139(3), 265-275.

Rajapaksha, I., Angus, P., \& Herath, C. (2019). Current therapies and novel approaches for biliary diseases. World Journal of Gastrointestinal Pathophysiology, 10(1), 1-10.

Rocha, F., Leite, A., Pompeu, M., Cunha, T., Verri, W. J., Soares, F., et al. (2008). Protective effect of an extract from Ascaris suum in experimental arthritis models. Infection and Immunity, 76(6), 2736-2745.

Sakaguchi, S. (2004). Naturally arising CD4+ regulatory T cells for immunologic self-tolerance and negative control of immune responses. Annual Review of Immunology, 22, 531-562.

Smits, H., van Rietschoten, J. G., Hilkens, C., Sayilir, R., Stiekema, F., Kapsenberg, M., et al. (2001). IL-12-induced reversal of human Th2 cells is accompanied by full restoration of IL-12 responsiveness and loss of GATA-3 expression. European Journal of Immunology, 31(4), 1055-1065.

Souza, V., Faquim-Mauro, E., \& Macedo, M. (2002). Extracts of Ascarissuumegg and adult worm share similar immunosuppressive properties. Brazilian Journal of Medical and Biological Research, 35(1), 81-89.

Sugiura, R., Ohnishi, S., Ohara, M., Ishikawa, M., Miyamoto, S., Onishi, R., et al. (2018). Effects of human amnion-derived mesenchymal stem cells and conditioned medium in rats with sclerosing cholangitis. American Journal of Translational Research, 10(7), 2102-2114.

Tacke, F. (2017). Targeting hepatic macrophages to treat liver diseases. Journal of Hepatology, 66(6), 1300-1312.

Terziroli, B.P. B., Guillod, C., Marsteller, I., Blum, R., Mazzucchelli, L., Mondino, C., et al. (2017). Primary biliary cholangitis associated with skin disorders: A case report and review of the literature. Archivum Immunologiae et Therapiae Experimentalis (Warsz), 65(4), 299-309.

Tjandra, K., Sharkey, K., \& Swain, M. (2000). Progressive development of a Th1-type hepatic cytokine profile in rats with experimental cholangitis. Hepatology, 31(2), 280-290.

Tsuchiya, K., Kirima, K., Yoshizumi, M., \& Tamaki, T. (2003). New methods to evaluate endothelial function: Evaluation of endothelial function by hemoglobin-nitric oxide complex using electron paramagnetic resonance spectroscopy. Journal of Pharmacological Sciences, 93(4), 417-422.

van Loo, G., Saelens, X., van Gurp, M., MacFarlane, M., Martin, S. J., \& Vandenabeele, P. (2002). The role of mitochondrial factors in apoptosis: A Russian roulette with more than one bullet. Cell Death and Differentiation, 9 1031-1042.

Woolbright, B. L., Antoine, D. J., Jenkins, R. E., Bajt, M. L., \& Park, B. K. (2013). Plasma bio- markers of liver injury and inflammation demonstrate a lack of apoptosis during obstructive cholestasis in mice. Toxicology and Applied Pharmacology, 273, 524-531

Yang, H., Ramani, K., \& Xia, M. (2009). Dysregulation of glutathionesynthesis during cholestasis in mice: Molecular mechanisms and therapeuticimplications. Hepatology, 49, 1982-1991.

Zaman, S., Wang, R., \& Gandh, V. (2014). Targeting the apoptosis pathway in hematologic malignancies. Leukemia \& Lymphoma, 55, 1980-1992.

\section{Publisher's Note}

Springer Nature remains neutral with regard to jurisdictional claims in published maps and institutional affiliations.

\section{Submit your manuscript to a SpringerOpen ${ }^{\odot}$ journal and benefit from:}

- Convenient online submission

- Rigorous peer review

- Open access: articles freely available online

High visibility within the field

- Retaining the copyright to your article

Submit your next manuscript at $\boldsymbol{\nabla}$ springeropen.com 